\title{
Droplet-based Microfluidic Device for the Synthesis of Silica Nanoparticles
}

\author{
Arsalan Nikdoost and E. Yegan Erdem \\ ${ }^{1}$ Mechanical Engineering Department, Bilkent University, Ankara, Turkey \\ *yeganerdem@bilkent.edu.tr \\ *Orcid No: 0000-0001-9852-2293
}

Received: 29 April 2020

Accepted: 14 September 2020

DOI: 10.18466/cbayarfbe. 729586

\begin{abstract}
Microfluidic reactors are advantageous for nanomaterial synthesis due to their capability to provide controlled reaction environment. In this work, a droplet based microfluidic reactor is designed for the synthesis of silica nanoparticles. The synthesis is carried out in a very controlled environment and a uniform size and shape distribution is achieved. The classical synthesis protocol for silica nanoparticles is modified to use nonpolar solvents in the reaction so that this platform can later be used for coating hydrophobic nanomaterials. Therefore, this study not only presents a new device but also a new synthesis method. The results are compared with conventional batch wise synthesis methods and the obtained nanoparticles showed better size distribution.
\end{abstract}

Keywords: microreactor, microfluidics, droplet-based flow, nanoparticles, silica nanoparticles, microfluidic synthesis

\section{Introduction}

Nanoparticles have unique electronic, optic, mechanic and biological properties along with their small size and high surface to volume ratio that make them desirable for applications in many areas such as drug delivery, printable electronics, wearable devices and sensor technologies. Silica nanoparticles are one type of them which attracted attention for their compatibility with other materials such as quantum dots and iron oxide that lead them to be desirable in optics and drug delivery [14]. Their properties are highly dependent on their size and morphology; therefore, their functionality increases when they are produced monodispersely.

Synthesis of nanoparticles are mostly done with conventional batch-wise methods where reagents are mixed and heated in large scales; however in these methods it is not always possible to maintain uniform reaction conditions in terms of time and temperature in all parts of the system, which results in polydisperse synthesis inevitably. Microfluidic synthesis methods are used to overcome this limitation as it is possible to control the reaction conditions such as residence time, concentration, temperature, and $\mathrm{pH}$ much precisely [5-8].

There are examples in literature that focus on the microfluidic synthesis of silica nanoparticles. For instance, the first sol-gel based microfluidic synthesis was performed by Khan et al. [9] where they used a segmented flow microfluidic device, with gas phase separating the liquid segments, and synthesized particles around $200-700 \mathrm{~nm}$. There were also other examples of microfluidic reactors which followed the well-known Stöber method where TEOS and ammonia solution is mixed [10-16]. In some of these reactors, flow was droplet-based where reagents were carried in the form of separate droplets in the channel [10-14, 17, 18] whereas some of them were continuous flow $[15,16]$. In most of these literature examples, though, the obtained particles were micron sized. Only a few of them targeted nanometer scale particles $[13,17]$.

In almost all these formerly presented work, reactants were prepared inside a polar solvent, mostly ethanol. This works very well if the synthesized particles are going to be used as is. However, for applications that require silica to be synthesized in situ with hydrophobic materials, such as in the case of coating of quantum dots with silica shells, a non-polar solvent is required during the synthesis [19]. Therefore, in this work a new microfluidic device was developed that can work with non-polar solvents in a droplet-based flow and compared to the results obtained by batch-wise synthesis, better size distribution with much reduced residence was achieved. 


\section{Materials and Methods}

\subsection{Fabrication of the Microreactor}

Microfluidic reactor is designed to have a droplet-based flow inside $200 \mu \mathrm{m}$ wide and deep channels. Two reagents are delivered into the microreactor by using capillary tubing, as soon as they enter the microchannel, a continuous flow separates them into individual droplets. Due to the circulating flow profile inside droplets, reagents mix rapidly without causing any clogging or contamination on channel walls. Dropletbased flow also maintains same amount of residence time as opposed to continuous flow scheme where the parabolic velocity profile causes an imbalance of total time spent in the channel. A schematic showing the layout of the microreactor is presented in Figure 1.

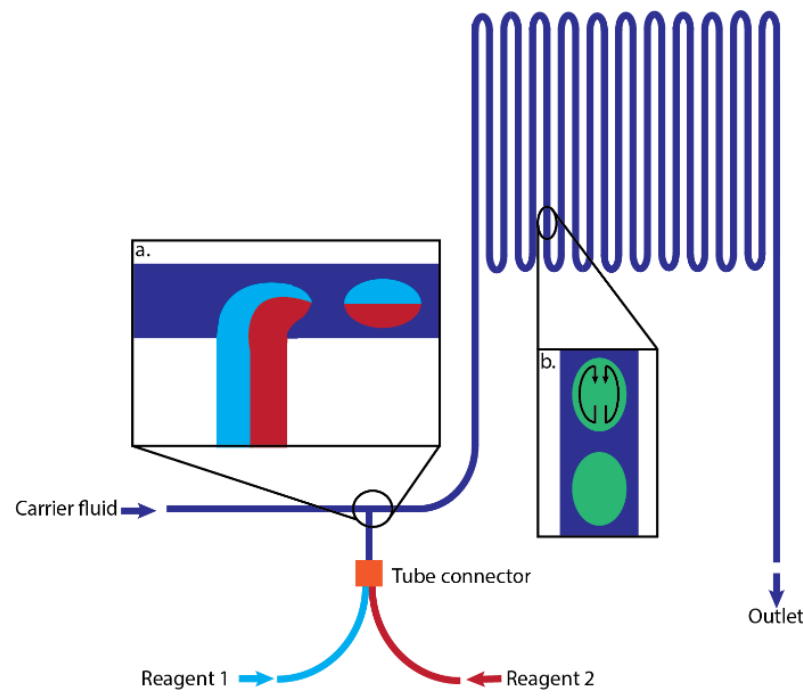

Figure 1. Schematic of the microfluidic device used for silica nanoparticle synthesis. (a) Droplet generation zone. (b) Schematic of the flow profile within droplets.

The fabrication of the microreactor was done in the clean room of National Nanotechnology Research Center (UNAM). The microchannels were etched with deep reactive ion etching in silicon wafer and later bonded to a glass substrate with anodic bonding. Later the capillary tubing is connected to the inlet of the reactor with epoxy. A tube connector is utilized to combine the flow incoming from two separate capillaries that carry two reagents and to deliver them to the microreactor.

\subsection{Synthesis Method}

The synthesis of silica nanoparticles with a nonpolar solvent was first performed by a batch-wise synthesis method in order to be able to compare the results obtained within the microfluidic reactor.

\subsubsection{Batch-wise Synthesis}

For the synthesis of silica nanoparticles compatible with hydrophobic coatings, a method proposed by Popovic et al. is adapted and modified [20]. A $1.3 \mathrm{~mL}$ IGEPAL CO-
520 and $10 \mathrm{~mL}$ of anhydrous cyclohexane were mixed. After the through mixing, $80 \mu \mathrm{L}$ of tetraethyl orthosilicate (TEOS) was added to this solution and stirred for 30 mins. Finally, $150 \mu \mathrm{L}$ of $28 \%$ ammonium hydroxide solution is added and stirred for 48 hours. Reaction was carried out at room temperature. Obtained silica nanoparticles were later centrifuged and dissolved in a $2 \mathrm{M} \mathrm{NaOH}$ solution and refrigerated. All solutions were obtained from Sigma Aldrich.

\subsubsection{Microfluidic Synthesis}

The microfluidic synthesis of silica nanoparticles was performed in a droplet-based flow where the reagents are carried within an immiscible flow as droplets. The selection of the carrier fluid is important as it should provide enough shear stress to form droplets and not mix with them. As the carrier fluid pure N-Methylformamide (NMF) was selected. Droplets were composed of two reagent solutions prepared separately and delivered to the microreactor from two separate capillary tubes. The first reagent solution was prepared with $1.3 \mathrm{ml}$ of IGEPAL CO-520 dissolved in $10 \mathrm{ml}$ of anhydrous cyclohexane and $80 \mu \mathrm{l}$ of TEOS. The second reagent solution was prepared by mixing $150 \mu \mathrm{l}$ of $28 \%$ aqueous ammonium hydroxide solution with $10 \mathrm{ml}$ of anhydrous cyclohexane. The choice of the carrier fluid was a challenge in here as a nonpolar solvent needed to be chosen for further processing with hydrophobic particles. Even though NMF is immiscible with cyclohexane and TEOS, it is slightly miscible with ammonium hydroxide. This might have resulted in loss of reactants from the droplets to the carrier fluid which would prevent the formation of particles. In order to avoid that, the concentration of the ammonium hydroxide solution in the droplets was determined experimentally.

Two reagent solutions were delivered to the microreactor from two separate capillary tubes, they were merged at a junction connected to the inlet and as soon as they entered the microchannel, the shear stress applied by the carrier fluid divided them into droplets. The two reagents were mixed inside droplets rapidly as they move in the channel.

\subsection{Experimental Set-up}

Experimental set-up for the microfluidic synthesis is composed of a microscope with camera attachment to monitor flow inside the channels, two syringe pumps for delivering liquids in the channel and a light source for imaging purposes.

\section{Results and Discussion}

In this work the synthesis was also carried with conventional batch methods to compare results obtained from the microreactor.

\subsection{Batch-wise Synthesis Results}

Batch-wise synthesis of silica nanoparticles lasted 48 hours as a result of the reverse micelle process explained earlier. The obtained nanoparticles were observed under 
the transmission electron microscope (TEM) and their size distribution is determined by looking at the images and calculating their diameter through direct measurement from these images. Particles were $18.25 \pm 2.2 \mathrm{~nm}$ in diameter. The TEM images are shown in Figure 2.

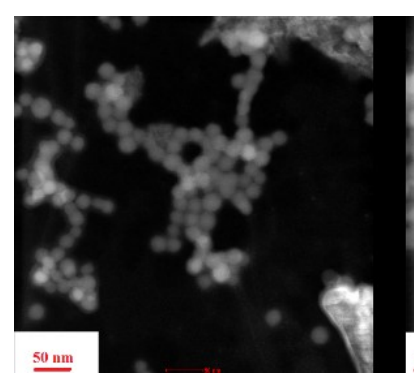

(a)

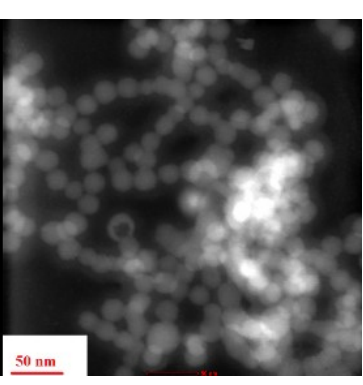

(b)
Figure 2. TEM images of synthesized silica nanoparticles in batch-wise method.

\subsection{Microreactor Synthesis Results}

For the synthesis of silica nanoparticles inside the microreactor, the flow rate of the continuous carrier fluid (NMF) was selected as $9.2 \mu \mathrm{l} / \mathrm{min}$ and the flow rates of two reagents (TEOS with cyclohexane and ammonium hydroxide with cyclohexane) were set as $1.9 \mu \mathrm{l} / \mathrm{min}$ for a stable droplet generation. Two reagents were delivered from separate capillary tubes as shown in Figure 3a. They merge with a microfluidic tube connector and enter the device, later form droplets (Figure $3 b$ ). Total residence time of reagents in the microreactor was 525 seconds and all experiments were carried out at room temperature.

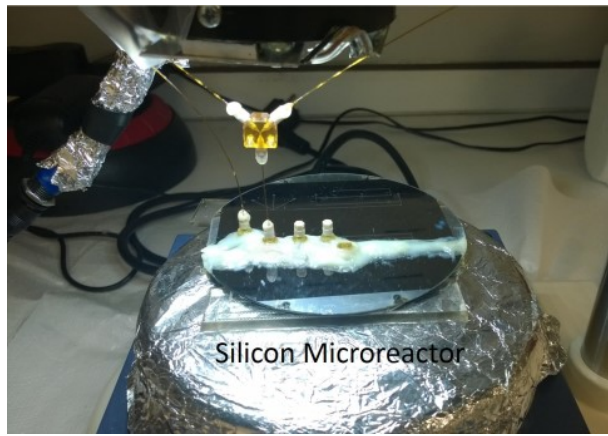

(a)
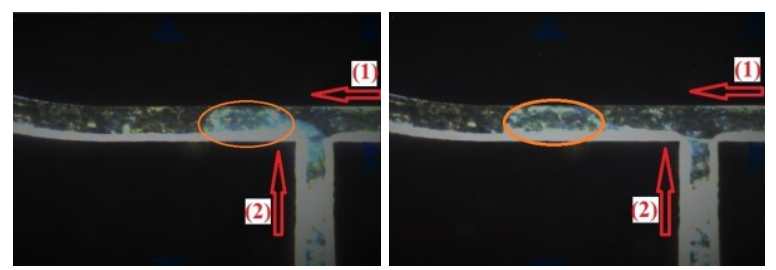

(b)

Figure 3. (a) The microreactor under operation. (b) Droplet formation inside the microchannel. (1) denotes the career fluid whereas (2) denotes the channel where droplet phase is delivered.
In the initial experiments, ammonium hydroxide in cyclohexane was $0.38 \mathrm{M}$ while the TEOS and cyclohexane solution was prepared as described in Section 2.2.2. The flow rate of the carrier fluid was $9.6 \mu \mathrm{l} / \mathrm{min}$ while TEOS solution was $0.8 \mu \mathrm{l} / \mathrm{min}$ and ammonium hydroxide solution with cyclohexane was $1.6 \mu \mathrm{l} / \mathrm{min}$. With this concentration and flow rates, the obtained silica nanoparticles were less than $4 \mathrm{~nm}$ in diameter, their image under the transmission electron microscope is shown in Figure 4.

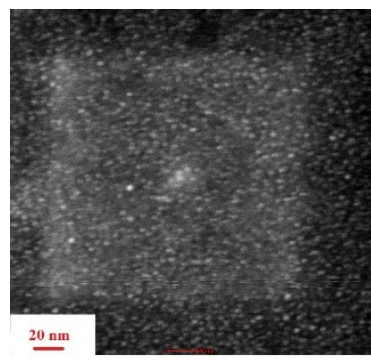

(a)

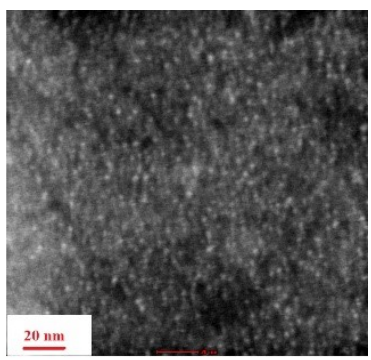

(b)
Figure 4. TEM images of silica nanoparticles synthesized in the microreactor with low ammonium concentration. The average diameter of the nanoparticles was less than $4 \mathrm{~nm}$.

The low ammonium concentration resulted in formation of seed like structures instead of individually distinguished nanoparticles. This may mean that the reagent was not sufficient for growth. In the next set of experiments, the ammonia solution concentration was doubled, and made $0.76 \mathrm{M}$ with a flow rate of $1.9 \mu \mathrm{l} / \mathrm{min}$. This gave resulted in silica nanoparticles with an average diameter of $25 \mathrm{~nm}$. The TEM images of these particles are shown in Figure 5. Particle sizes were measured by using Adobe Illustrator program with manual measurement of diameter of each particle in the images.

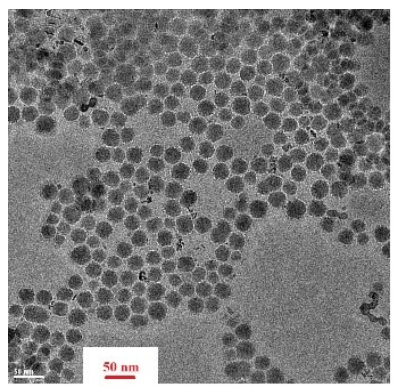

(a)

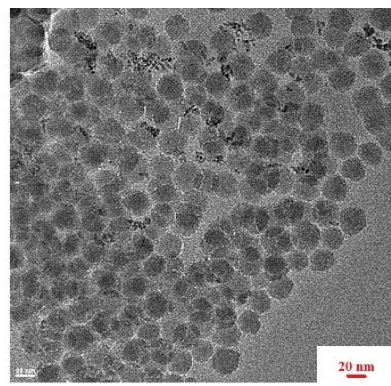

(b) 


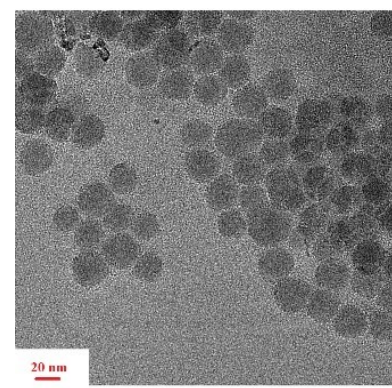

(c)

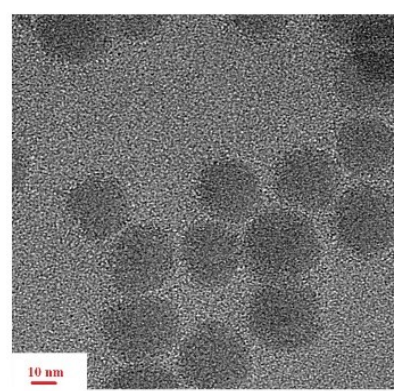

(d)
Figure 5. TEM images of nanoparticles synthesized within the microreactor with high ammonium concentration. Average diameter size is $25 \pm 2.7 \mathrm{~nm}$.

In order to confirm the formation of silica nanoparticles, EDX analysis was performed on samples obtained from the microreactor. As a sample holder a TEM grid was used due to the small amount of nanoparticle solution. Figure 6 shows the EDX results. Since the TEM grid was made from $\mathrm{Cu}, \mathrm{Cu}$ peaks also appeared along with $\mathrm{Si}$ and O peaks.

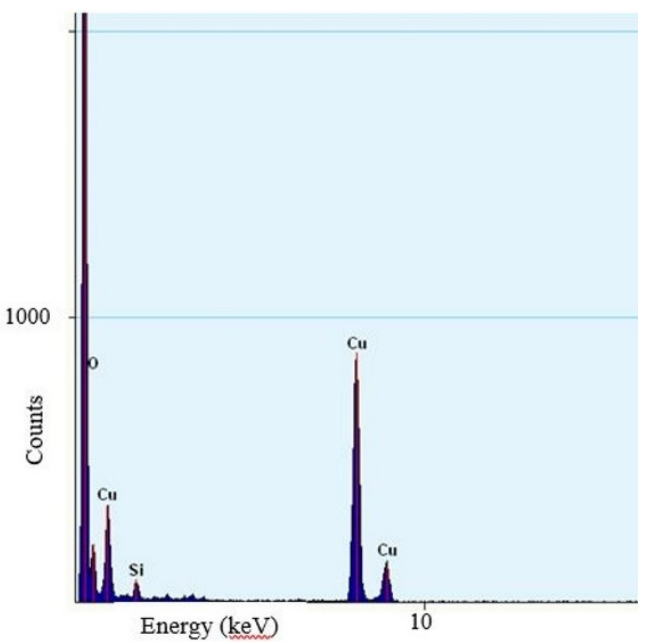

Figure 6. EDX quantification results.

\subsection{Comparison of the Results}

As a final step the results obtained from batch synthesis and microfluidic synthesis were compared. The major difference is the reaction time and the ammonium hydroxide concentration. In the batch-wise synthesis method, the reaction lasted 48 hours and the ammonium hydroxide concentration was $0.38 \mathrm{M}$. The resulting nanoparticles were $18.25 \pm 2.7 \mathrm{~nm}$ in diameter. In the microfluidic synthesis, the ammonium hydroxide concentration was doubled $(0.76 \mathrm{M})$ while the reaction lasted for $8.75 \mathrm{~min}$. The obtained nanoparticles were $25 \pm 2.7 \mathrm{~nm}$ in diameter. This shows that as the reagent amounts are decreased and reaction is carried out in microenvironments, the residence time decreases while the size distribution is improved. This confirms the advantage of using microfluidic reactors in nanomaterial synthesis.

\section{Conclusion}

A novel microfluidic device is designed for the synthesis of silica nanoparticles with a nonpolar solvent. A dropletbased flow is utilized to control reagent concentrations precisely and to obtain a uniform mixing. The synthesis was successful and resulted in a narrower size distribution compared to the batch-wise methods. In addition, the synthesis was accomplished in shorter times than available methods in literature. This platform and synthesis method can later be used for coating of quantum dots with silica for increasing their stability.

\section{Acknowledgement}

E. Y. Erdem would like to acknowledge the Tübitak 2232 grant that funded this research under the project number 114C092. She also thanks Mr. Alican Özkan for their help in the laboratory studies as well as Dr. Yusuf Keleştemur for assisting with imaging and providing some of the chemicals used in the reaction.

\section{Author Contributions}

Arsalan Nikdoost fabricated the microreactor and performed the experimental work.

E. Yegan Erdem designed the microreactor and experimental steps. Both authors contributed in the writing and reviewing of the manuscript.

\section{Ethics}

There are no ethical issues after the publication of this manuscript.

\section{References}

1. He, Q, Shi, J. 2010. Mesoporous silica nanoparticle based nano drug delivery systems: synthesis, controlled drug release and delivery, pharmacokinetics and biocompatibility. Journal of Materials Chemistry; 21: 5845-5855.

2. Zhao, CX, He, L, Qiao, SZ, Middelberg, APJ. 2011. Nanoparticle synthesis in microreactors. Chemical Engineering Science; 66(7): 1463-1479.

3. $\mathrm{Wu}, \mathrm{KCW}$, Yamauchi, Y. 2012. Controlling physical features of mesoporoussilica nanoparticles (MSNs) for emergingapplications. Journal of Materials Chemistry; 22:12511256.

4. Mebert, AM, Baglole, CJ, Desimone, MF, Maysinger, Dusica. 2017. Nanoengineered silica: properties, applications and toxicity. Food and Chemical Toxicology; 109(1): 753-770.

5. Erdem, EY, Cheng, JC, Doyle, FM, Pisano, AP. 2014. Multitemperature zone, droplet-based microreactor for increased temperature control in nanoparticle synthesis. Small; 10(6): 10761080 . 
6. Phillips, TW, Lignos, IG, Maceiczyk, RM, DeMello, AJ, DeMello, A. 2014. Nanocrystal synthesis in microfluidic reactors: where next?. Lab Chip.; 14(17): 3172-3180,

7. Chang, CH, Paul, BK, Remcho, VT, Atre, S, Hutchison, JE. 2008. Synthesis and post-processing of nanomaterials using microreaction technology. Journal of Nanoparticle Research; 10 965-980.

8. Nightingale, AM, deMello, JC. 2012. Segmented flow reactors for nanocrystal synthesis. Advanced Materials; 25(13): 1813-1821.

9. Khan, SA, Gunther, A, Schmidt, MA, Jensen, KF. 2004 Microfluidic synthesis of colloidal silica. Langmuir; 20(20): 8604-8611.

10. Lee, I, Yoo, Y, Cheng, Z, Jeong, HK. 2008. Generation of monodisperse mesoporous silica microspheres with controllable size and surface morphology in a microfluidic device. Advanced Functional Materials, 18(24): 4014-4021.

11. Carroll, NJ, Rathod, SB, Derbins, E, Mendez, S, Weitz, DA, Petsev, DN. 2008. Droplet-based microfluidics for emulsion and solvent evaporation synthesis of monodisperse mesoporous silica microspheres. Langmuir; 24(3): 658-661.

12. Li, D, Guan, Z, Zhang, W, Zhou, X, Zhang, WY, Zhuang, Z, Wang, X, Yang, CJ. 2010. Synthesis of Uniform-Size Hollow Silica Microspheres through Interfacial Polymerization in Monodisperse Water-in-Oil Droplets. ACS Appl. Mater. Interfaces; 2(10): 2711-2714.

13. Su, M, Su, H, Du, B, Li, X, Ren, G, Wang, S. 2014. The propertie of silica nanoparticles with high monodispersity synthesized in the microreactor system. Journal of Sol-Gel Science and Technology; 72(2): 375-384.

14. Yan, H, Kim, C. 2014. Formation of monodisperse silica microparticles with various shapes and surface morphologies using double emulsion templates. Colloids and Surfaces A: Physicochemical and Engineering Aspects; 443(20): 88-95.

15. He, Y, Kim, KJ, Chang, CH. 2017. Continuous, size and shapecontrol synthesis of hollow silica nanoparticles enabled by a microreactor-assisted rapid mixing process. Nanotechnology; 28(23): 235602

16. Hao, N, Nie, Y. Xu, Z, Closson, AB, Usherwood, T, Zhang, JXJ 2019. Microfluidic continuous flow synthesis of functional hollow spherical silica with hierarchical sponge-like large porous shell. Chemical Engineering Journal; 366: 433-438.

17. He, P, Greenway, G. Haswell, SJ. 2011. Microfluidic synthesis of silica nanoparticles using polyethylenimine polymers. Chemical Engineering Journal; 167(2): 694-699.

18. Wacker, JB, Lignos, I, Parashar, VK, Gijs, MAM. 2012 Controlled synthesis of fluorescent silica nanoparticles inside microfluidic droplet. Lab Chip; 12: 3111-3116.

19. Kobayashi, Y, Nozawa, T, Nakagawa, T, Gonda, K, Takeda, M, Ohuchi, N, Kasuya, A. 2010. Direct coating of quantum dots with silica shell. Journal of Sol-Gel Science and Technology; 55: 7985 .

20. Popović, Z, Liu, W, Chauhan, VP, Lee, J, Wong, C, Greytak, AB Insin, N, Nocera, DG, Fukumura, D, Jain, RK, Bawendi, MG. 2010. A nanoparticle size series for in vivo fluorescence imaging. Angewandte Chemie; 122(46): 8831-8834. 